\title{
Understanding internet use and dependency
}

\author{
Ch.-H. Soh \& R Murali \\ Faculty of Management, Multimedia University, Cyberjaya, Malaysia
}

\begin{abstract}
Since the 1950s, the Uses and Gratifications approach has been widely applied to explaining the use of the media, such as the radio and newspapers. Recently, this framework has also been applied to the Internet, a new media and has helped in the understanding of how the motivations of individuals affect their Internet usage patterns and their consequences.

The Internet is arguably very different from the conventional media, with salient features like interactivity, anonymity, chameleon of features, rich multimedia, censorship, the wide breadth and depth of information provided. More importantly, the Internet is a medium used mostly by the young and hence, the role of supervision should perhaps not be ignored. However, only limited, if any empirical studies on the role of supervision on internet usage and dependency. On the other hand, studies that have been conducted on parental supervision and adolescent behaviour such as delinquency, alcohol and drug use and these have concluded that parental supervision is an important factor in deterring deviant adolescent behaviour.

This paper proposes a modification of the widely supported Uses and Gratification framework to better understand Internet usage and the dependency of adolescents. The proposed framework could help raise the awareness on risk mitigation on the Internet.
\end{abstract}

Keywords: Internet, addiction, youth, parental supervision, deviant behaviour.

\section{Introduction}

Since the 1950s, the Uses and Gratifications approach has been widely applied to explaining the use of the media, such as the radio and newspaper. Recently, this framework has also been applied to the Internet, a new media and has helped in the understanding of how the motivations of individuals affect their Internet usage patterns and their consequences. Many empirical studies use this 
framework to understand the motives and psychological factors of individuals e.g. loneliness, depression, self-control and correlate them to their usage and dependence of the Internet [1-4].

The question arises as to whether the Internet can be treated the same as conventional media such as radio, television and the newspaper. The Internet is arguably very different from the conventional media, with salient features like interactivity, anonymity, chameleon of features, rich multimedia, lack of censorship, the wide breadth and depth of information provided.

More importantly, the Internet is a medium used mostly by the young and hence, the role of supervision should perhaps not be ignored. Nevertheless, there have been few, if any, empirical studies on the role of supervision on internet usage and dependency. On the other hand, studies have been conducted on parental supervision and adolescent behaviour such as delinquency, alcohol and drug use have concluded that parental supervision is an important factor in deterring deviant adolescent behaviour [5-7].

This paper proposes a modification of the widely supported Uses and Gratification framework [8] for a better understanding of Internet usage and dependency by adolescents. The proposed framework can help raise awareness on risk mitigation on the Internet.

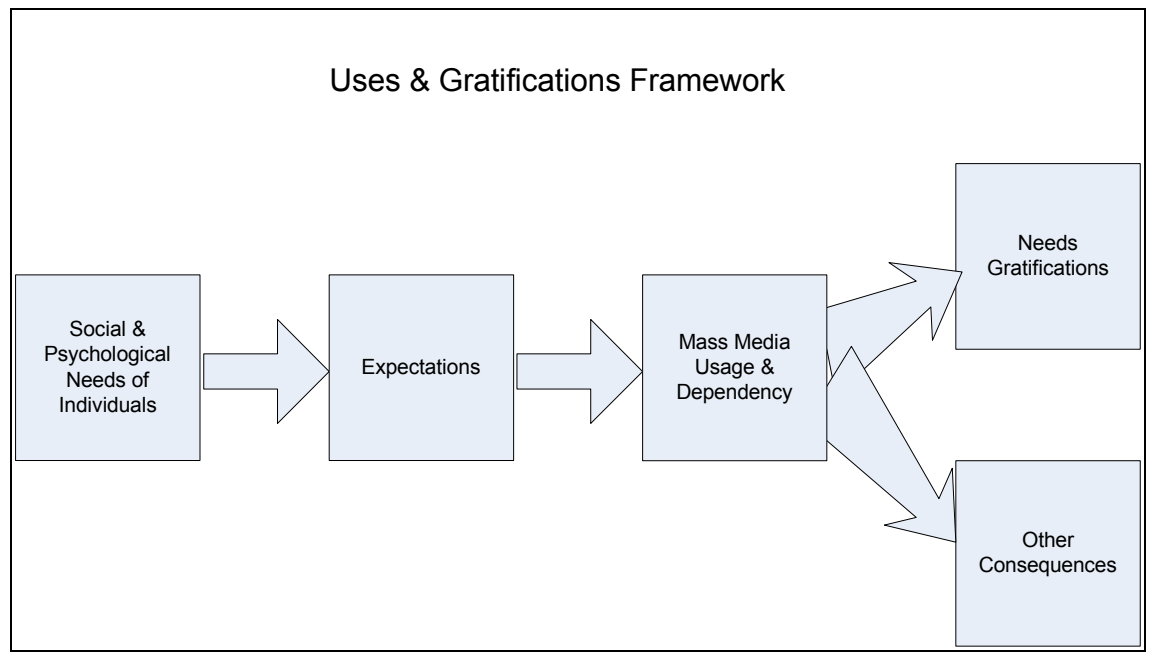

Figure 1: $\quad$ Uses and gratifications framework [8].

\section{Uses and gratifications framework}

The Uses and Gratifications framework originated from the 1940s when researchers wanted to understand the motivations of people using media such as reading the newspapers or listening to the radio. The approach is based on the concept that people choose the media according to their needs and the gratifications they expect the media to provide. Katz [8] wanted to find out what 
people do with the media, how they use it and which needs the media fulfilled. Katz's approach was built on the premise that the audience of the media was not passive, but active and selective in the communication process. The framework for the uses and gratifications are:

a) To explain how people use the media to meet their needs.

b) To understand the motives for their dependence on the media.

c) To identify the consequences that resulted from the needs, motives and dependence.

Figure 1 illustrates the framework for the Uses and Gratification approach.

The Uses and Gratifications approach has also been popularly applied to the Internet [1-4] to study its usage and dependency. Among the numerous studies carried out, Choi [4] investigated the motivations and internet use patterns of 355 Koreans based on the uses and gratification approach. The results indicated that Koreans use the Internet for entertainment, sexuality, online transactions and social interactions. Their usage of the Internet reduces their utilization of other media sources. Huang [2] found that the motives of the $\mathrm{N}$-generation (13-24 years old) Taiwanese for using the Internet are entertainment, escape, surveillance, convenience and social interactions. Salience among these motives is entertainment and escape. The average Internet use was 2.5 hours daily and 15.56 hours weekly. One third of the respondents were classified as Internet addicts and they spend 20-32 hours online each week, particularly on online interactive games (97\%). Park [9] studied the Internet usage of 377 Korean and American tertiary students in the United States. Factor analysis revealed that the Americans and Korean students share six common gratification dimensions, which are social communication, information surveillance, pastime escape, download entertainment, personal communication and research. An additional gratification of the American students, but not by the Korean students is online transaction. There were other clear differences of usage between the two groups of students. The two top gratifications for American students are social communication and information-surveillance, while for Korean students are pasttime escape and download entertainment.

\section{The unique features and risks on the Internet}

There is little doubt that the Internet has been widely accepted and used throughout the world. Its unique strengths such as its ubiquity, lack of censorship, chameleon of features and interactivity had contributed to its immense popularity. The ubiquity of the Internet allows children to access the Internet at any hours from the privacy and safety of their homes, often with their parents thinking their children are safe at home and doing their homework. This is unlike any conventional addiction, where parents can protect their children simply by disallowing them to be exposed to risky places and people such as arcades or gambling dens through confining their children at homes. 
The lack of censorship of the contents on the Internet has lead to online gambling, pornography not only to be widely accessible but also often aggressively promoted to unsuspecting children $[10,11]$. The anonymity afforded by the Internet has allowed the curious to "look into" forbidden sites and sights, and participate in risky online behaviour like cybersex and flirting $[10,11]$. Rich multi-media interactivity allows paedophiles to target and entrap innocent children in the safety of their homes[10].

The Internet is a medium that is mostly used by youth, rather than adults [12] and its usage is increasing every year. Current research has indicated that youth face risks on the Internet, such as dependence, exposure to unsolicited pornography and sexual solicitation [13]. The sexual exploitation of children via the Internet has become so prevalent to be classified as a "serious problem" [14]. A nationally representative sample of 1,501 surveyed children in the United States has found that about $25 \%$ of them are exposed to unwanted sexual materials. Also, $19 \%$ of them were sexually propositioned while online and more than a third of them did not report this to adults [13]. A UK study found that $20 \%$ children aged under 17 had visited pornographic web sites [15]. In addition to sexually explicit materials, the young may visit fictional and documentary violence sites including bomb-making instructions, hate propaganda, pictures of suicide, and instructions on suicide $[16,17]$.

There has been a number of studies indicating unhealthy dependence on the Internet, even to the point of being classified as an addiction [1, 2, 18, 19]. Empirical studies have shown that addiction to the Internet has led to online affairs [20], neglect of work [20-22] and social life [21]. As addicts become unable to limit their online hours and fail to turn up for work or misuse their office computer facilities, they could even loss their jobs [23]. Adverse effects on the person's emotions, social life, work and finances have been documented for compulsive cybersex participants [24].

Students are possibly the most at-risk population because of encouraged use in their campuses compounded by their unstructured time blocks [20, 25, 26]. According to researchers, approximately 5-10\% of college students suffer adverse effects like craving, sleep disturbance, depression and even withdrawal symptoms of excessive online hours. Scherer [27] and Anderson [28] have discovered that $13 \%$ and $9.8 \%$ college students respectively were Internet dependent. A study by Young $[20,22]$ found that $58 \%$ of students suffered from poor study habits, poor grades or failed school because of excessive Internet use. Similarly, a survey of 527 students at a large public university in America have strongly linked academic problems with excessive Internet use [29].

Some colleges have become aware of the relatively small, but growing problem of Internet dependence. A study reveals that $86 \%$ of responding educators believe that Internet usage by children does not improve performance and can even serve as a distraction [30]. Alfred University, who dismissed normally successful students with 1200 to 1300 SATs, was surprised to find that their extensive late night logins patterns was a major factor in their academic decline [31]. William Woods University in Missouri has instituted a new 
program that were designed to reputably encourage students to move offline [29].

\section{Supervision of Internet usage}

Supervision of Internet usage can include parental supervision, teacher guidance, use of blocking, filtering software and sites that require a credit card number to gain access. Apart from parental supervision and filtering software, the other measures are still at their infancy. It would seems that the primary responsibility of preventing children to access offensive material has been left to parents, as made clear by the United States courts [32]. Yet, there has been little research on parental supervision and use of filtering software on the Internet. Unlike other media, children and teenagers are often much more knowledgeable and skilful with the Internet than their parents [33]. Parents believe that computers are the way of the future, and often encourage their children to "use" the Internet.

According to Young [22], parents/caregivers practice either 'benign neglect' of their children's Internet usage or 'outright banishment'. Research indicates there is little adult supervision of the youth's Internet usage in the United States [10]. Interestingly, virtually all the individual case studies of adolescent Internet addicts cited by Young [22] accessed the Internet in their bedrooms without adult supervision. This situation is likely to be worse in many developing countries like Malaysia where adults can be illiterate and are often ignorant and fearful of this new media. Interestingly, there is a significant discrepancy between attitudes towards monitoring Internet and restricting television viewing. Research in Australia found that $98 \%$ of parents restrict their children's television viewing [34] but only a small number monitor internet usage of their children.

One of the alternatives for parents is to install filtering and blocking software. In a survey, Mitchell [35] reported that slightly more than one out of 3 families in America installed filtering and blocking software. However, no software package is going to be $100 \%$ effective in blocking out unacceptable contents in the continuously changing online environment. Such software can be overly restrictive and unintentionally block legitimate sites. A small scale study of four popular filtering software showed that they failed to block $25 \%$ of objectionable sites, while also blocking $21 \%$ of no objectionable test sites [36]. Richardson [15] found that the most restrictive block setting blocked $91 \%$ of pornography and $24 \%$ of health information. Mitchell [35] indicated that using such software seems to reduce by $40 \%$ unwanted exposure to sexual materials.

Unlike the paucity of research into supervision of online behaviour, there have been many studies on the impact of parental supervision on deviant adolescent behaviour such as delinquency, alcohol use and drug use. These studies have concluded that parental supervision does play an important factor in deterring deviant adolescent behaviour [5-7]. Parental supervision refers to establishing rules and limits to be observed by children, and includes the quantity and accuracy of the information of the parents about their adolescents' daily life. Inadequate supervision may be characterized by absence of control and laxness, 
presence of permissiveness and inability to set limits and has been strongly correlated with poor academic performance [37], delinquency [6,38] or drug use[7, 39]. This strong correlation existed across different cultures and countries as affirmed by Claes [7], who did a study confirming that parental supervision influences deviant late adolescent behaviour across 3 different countries, Canada, France and Italy. Indeed, Berson's online study of adolescent girls in the United States [10] highlights that the influence of parents and teachers may assist students to avoid harmful objectionable activities.

Based on the preceding discussion, it would seem logical to revise the Uses and Gratifications framework (Figure 2.) More importantly would be to study the role and impact of supervision on the Internet usage of adolescents. If there is little supervision as indicated by some studies [10,22], then perhaps it is timely to raise the awareness of the need for supervision of online behaviour.

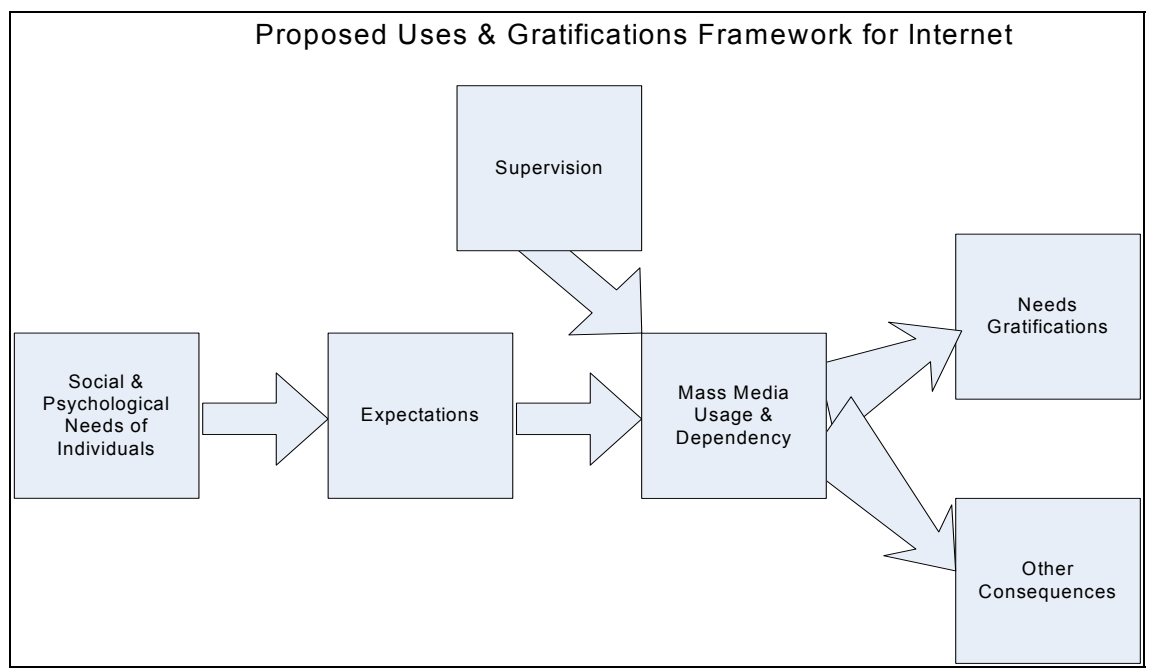

Figure 2: $\quad$ Revised framework for Internet use.

\section{Measuring supervision}

If we should measure the extent of supervision on the Internet, how do we go about doing it? A suggestion would be to ask the following questions:

1. Do parents use the Internet?

2. Do parents know about the web sites and online activities they participate?

3. Do parents issue guidelines on the length of hours the adolescent can go online?

4. Do they use filtering and blocking software in their computers?

5. Is the computer situated in their bedrooms or common areas?

6. Do their colleges or schools issue guidelines on Internet usage? 
Answers to the question could be in a range of 1 to 5 , with 1 being the lowest and 5 the highest/most frequent. The first three questions pertain to the direct supervision of parents. The first question presupposes that parents who know how to use the Internet could be better aware of the dangers and could possibly better guide or supervise their children. Questions 4 and 5 relate to indirect control of the parents. Question 6 attempts to find out whether schools, colleges or computer clubs issue practical guidelines on using the Internet.

The questions attempt to find out whether the degree of supervision. This could then be correlated with the number of online hours spend for recreational usage (as opposed to using the computer for academic purposes).

An interesting question to gauge the difference in perceptions of parents towards the Internet and television would also be to ask:

- Do parents issue guidelines on the number of hours the children can watch television?

- Do parents limit their children to the type of television programs can watch?

Possibly, some questions could be asked about the risky online behaviour of the adolescents. These could be:

- Do adolescent visit any objectionable websites e.g. pornographic, violent sites?

- Do adolescent engage in online chat with strangers?

- Do adolescent engage in sexually oriented online chat?

- Have any of their online friends (whom you have never met) asked to meet with them?

This paper is part of an ongoing doctoral study. The next steps for this study are to design a survey, carry out a nation wide survey of the youth in Malaysia, and to find the degree of parental supervision and the correlation between supervision, Internet usage and dependency.

\section{References}

[1] Charney, T.R. and B.S. Greenberg, Uses and Gratifications on the Internet. Communication, technology and society: New Media Adoption and Uses, ed. A.L.D.J. Atkin. 2001, Cresskill, NJ: Hampton Press. 397-407.

[2] Huang, A.S.C., The Bright and Dark Side of Cyberspace: The paradoxical media effects of internet use on gratifications, addictions, social and psychological well-being among Taiwan's Net-Generation., in School of Journalism. 2004, Southern Illinois University: Carbondale. p. 188.

[3] Song, I., Internet Gratifications, Depression, Self-Efficacy and Internet Addiction, in Department of Telecommunications. 2003, Michigan State University. p. 198. 
[4] Choi, Y.J., Investigating Koreans' internet use patterns and motivations, and exploring vulnerability of internet dependency, in Graduate School. 2001, The University of Southern Mississippi. p. 166.

[5] Barreta, M., et al., Replication of a problem behaviour model with American Indians, Hispanic and Caucasian youth. Journal of Early Adolescence, 2001. 21: p. 133-157.

[6] Herman, M.R., et al., The influence of family regulation, connection and psychological autonomy on six measures of adolescent functioning. Journal of Early Adolescence, 1997. 12: p. 34-67.

[7] Claes, M., et al., Parenting, Peer Orientation, Drug Use, and Antisocial Behaviour in Late Adolescence: A Cross-National Study. Journal of Youth and Adolescence, 2005. 34(5): p. 401 - 411.

[8] Katz, E., Mass communication research and the study of popular culture: An editorial note on a possible future for this journal. Studies in Public Communication, 1959. 2: p. 1-6.

[9] Park, I., Internet Usage of Korean and American Students- A Uses and Gratifications Approach, in College of Arts and Letters. 2004, University of Southern Mississippi. p. 169.

[10] Berson, I.R., M.J. Berson, and J.M. Ferron, Emerging Risks of Violence in the digital age: Lessons for Educators from an Online Study of Adolescent Girls in the United States. Journal of School Violence, forthcoming.

[11] Elmer-DeWitt, P., On a screen near you: Cyberporn - It's popular, pervasive and surprisingly perverse, in Time. 1995. p. 38 - 45.

[12] Nie, N.H. and L. Erbring, Internet and society, Q.S.o. Society, Editor. 2000, Stanford University: Stanford, CA.

[13] Finkelhor, D., K.J. Mitchell, and J. Wolak, On-line victimisation: a report on the nations' youth. 2000, National Centre for Missing and Exploited Children, 699 Prince Street, Alexandra VA: Alexandra VA.

[14] EPCAT_(End_Child_Prostitution_and_Trafficking). 2001.

[15] Richardson, Does Pornography-Blocking Software Block Access to Health Information on the Internet? Journal of American Medical Association, 2002. 288: p. 2887-2894.

[16] Carr, J., An innocent encounter, in Professional Social Work. 2001. p. 12-13.

[17] Feather, M. Internet and child victimisation. in Children and Crime: Victims and Offenders Conference. 1999. Brisbane.

[18] Young, K.S., Internet Addiction: The emergence of a new clinical disorder. Cyber Psychology \& Behaviour, 1996. 1(3): p. 237-244.

[19] Bullen, P. and N. Harre, The Internet: Its Effects on Safety \& Behaviour: Implications for Adolescents, D.o. Psychology, Editor. 2000, University of Auckland: Auckland. p. 20.

[20] Young, K.S., Internet Addiction: A New Clinical Phenomenon and Its Consequences. The American Behavioral Scientists, 2004. 48(4): p. $402-415$.

[21] Winyanto, L. and M. McMurran, The Psychometric Properties of the Internet Addiction. Cyber Psychology \& Behavior, 2004. 7(4): p. 2004. 
[22] Young, K.S., Caught in the net: how to recognise the signs of Internet addiction and a winning strategy for recovery. 1998, New York: John Wiley \& Sons, Inc.

[23] Orzack, M.H., Computer Addiction Services. 2003.

[24] Schneider, J.P., M.D., A Qualitative Study of Cybersex Participants: Gender Differences, Recovery Issues and Implications for Therapists. Sexual Addiction \& Compulsivity, 2000. 7: p. 249-278.

[25] Young, J.R., Students are unusually vulnerable to Internet Addiction, Article Says. The Chronicle of Higher Education, 1998. 44(22): p. A25.

[26] Finkellior, D., K.J. Mitchell, and K. Wood, Highlights of the Youth Internet Safety Survey. 2001, U.S. Department of Justice. p. 2.

[27] Scherer, K. College life online: Healthy and unhealthy Internet use. in 104th Annual Meeting of the American Psychological Association. 1997. Chicago.

[28] Anderson, K. Internet use among college students: Should we be concerned? in American Psychological Association. 1999. Boston, MA.

[29] Kubey, W.R., J.M. Lavin, and J.R. Barrows, Internet Use and Collegiate Academic Performance Decrements: Early Findings. Journal of Communication, 2001. 51(2): p. 366 - 382.

[30] Barber, A., Net's educational value questioned., in USA Today. 1997. p. 4D.

[31] Brady, K., Dropout rise a net result of computers, in The Buffalo News. 1996. p. A1.

[32] Ferrier, M., Censorship, 'cookies' and milk: Children and protections on the internet. 2000.

[33] Technology, U.S.T.a., U.S. teens and technology survey. 1997.

[34] Durkin, K. and J. Low, Children, media and aggression: Current Research in Australia and New Zealand, in Children and Media Violence, U.C.C.v. Feilitzen, Editor. 1998, The UNESCO International Clearinghouse on Children and Violence on the Screen: Goteborg. p. 107124.

[35] Mitchell, K.J., D. Finkellior, and K. Wood, The Exposure of Youth to Unwanted Sexual Material on the Internet: A National Survey of Risk, Impact, and Prevention. Youth \& Society, 2003. 34(3): p. 330 - 358.

[36] Hunter, C.D., Internet filter effectiveness: Testing over and underinclusive blocking decisions of four popular filters. Social Science Computer Review, 2000. 18(2): p. 214 - 222.

[37] Dornbush, S.M. and K. Wood, Family processes and education achievement. Education and the American Family: A Research Synthesis, ed. W.J. Weston. 1989, New York: New York University Press.

[38] Lamborn, S.D., et al., Patterns of competence and adjustment among adolescents from authoritative, authoritarian, indulgent and neglectful families. Children Development, 1991. 62: p. 1049-1065.

[39] Loeber, R. and T.J. Dishion, Early predictors of male delinquency: $A$ review. Psychology Bulletin, 1983. 94: p. 68 - 99. 